AperTO - Archivio Istituzionale Open Access dell'Università di Torino

\title{
A Multi-UAS Cooperative Mission Over Non-Segregated Civil Areas
}

\section{This is the author's manuscript}

Original Citation:

Availability:

This version is available http://hdl.handle.net/2318/120872

since 2016-06-29T12:09:21Z

Published version:

DOI:10.1007/s10846-012-9706-5

Terms of use:

Open Access

Anyone can freely access the full text of works made available as "Open Access". Works made available under a Creative Commons license can be used according to the terms and conditions of said license. Use of all other works requires consent of the right holder (author or publisher) if not exempted from copyright protection by the applicable law. 


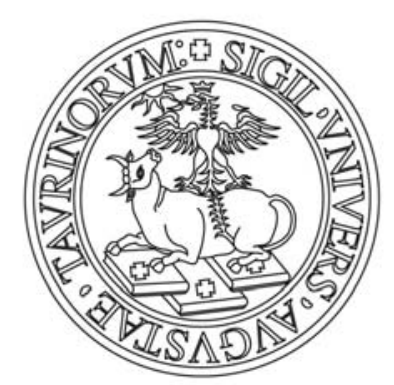

\section{UNIVERSITÀ DEGLI STUDI DI TORINO}

This is an author version of the contribution published on:

Questa è la versione dell'autore dell'opera:

Marco Boccalatte · Filippo Brogi · Francesco Catalfamo - Stefania Maddaluno Michele

Martino · Valter Mellano · Paolo Rosazza Prin · Filomena Solitro ·

Pietro Torasso - Gianluca Torta :

A Multi-UAS Cooperative Mission Over Non-Segregated Civil Areas,

Journal of Intelligent and Robotic Systems (2013) 70:275-291

DOI 10.1007/s10846-012-9706-5

The definitive version is available at:

La versione definitiva è disponibile alla URL:

http://link.springer.com/article/10.1007\%2Fs10846-012-9706-5 


\title{
A Multi-UAS Cooperative Mission over non-Segregated Civil Areas*
}

\author{
M. Boccalatte, F. Brogi, F. Catalfamo, S. Maddaluno, M. Martino, V. Mellano, \\ P. Rosazza Prin, F. Solitro, P. Torasso and G. Torta
}

\begin{abstract}
In this paper we describe an experience of multi UAS civil mission management derived from our participation to the Industrial Research Project SMAT-F1, which focused on UAV missions for monitoring the territory for civil purposes. After describing the operational framework and the system architecture, we present in some detail the computer-supported design of a Mission Plan. The main focus of the article is on the flight experience made; in particular, we discuss the capabilities of the system to supply clear situation comprehension to the operators, the operation coordination issues and the operational results achieved during the mission. Finally, future extensions of mission planning support capabilities are discussed.
\end{abstract}

\section{INTRODUCTION}

The SMAT-F1 project (funded by the Regione Piemonte - Italy, and co-funded by the European fund for regional development) was motivated by new requirements of cost reduction, increased capabilities and performances that are maturing in the latest years in the wide domain of surveillance via Unmanned Air Systems (UAS). SMAT-F1 is addressing also the evolution of technologies of unmanned aircraft that are assuming an important role in aeronautics and that are leading to a new class of highly reliable products.

SMAT-F1 represents the point of contact between the increasing need of Governmental Agencies and private organizations of a land and environment monitoring system and the technology evolution in Unmanned Air Systems (UAS). As a matter of fact, Unmanned Air Vehicles (UAV) can been employed in so called DDD missions (Dull, Dirty, and Dangerous) and in routine missions, that require a long flight endurance. Ordinary and extraordinary land monitoring are mission typologies with these peculiarities and in addition UAS life-cycle costs are smaller than the costs of comparable manned aircraft.

In recent years the interest for cooperative missions involving multiple UAS has rapidly increased and has moved from military applications to cover also civil applications (see, e.g., [1]).

\footnotetext{
* Research supported by the European fund for regional development (F.E.S.R.) within the Regional Operative Program 2007/2013. SMAT-F1 started on January 2009 and the project working group, led by Alenia Aermacchi, is composed by: three large industries: Alenia Aermacchi, Selex Galileo, ALTEC; two universities: Politecnico di Torino, Università di Torino; one Research Centre: Istituto Superiore Mario Boella; and eleven Small Medium Enterprises (SME) operating in the Regione Piemonte: Auconel, Axis, Blue Engineering, DigiSky, Envisens Techonologies, Italia Design Torino, Nautilus, Nimbus, SEPA, SPAIC, Synarea.

F. Brogi and S. Maddaluno are with Alenia Aermacchi, A Finmeccanica Company, C.so Marche, 41 - 10146 Torino (TO) - Italy (e-mail: name.surname@alenia.it).

M. Martino, P. Rosazza Prin and F. Solitro are with ALTEC S.p.A., C.so Marche, 79 - 10146 Torino - (Italy) (e-mail: name.surname@altecspace.it).

M. Boccalatte, F. Catalfamo and V. Mellano are with the Selex Galileo, A Finmeccanica Company, Strada Privata Aeroporto di Caselle - 10077 S. Maurizio C.se (TO) - (Italy) (e-mail: name.surname@selexgalileo.com).

P. Torasso and G. Torta are with Dipartimento di Informatica, University of Turin, C.So Svizzera, 185 - 10149 Torino - (Italy) (e-mail: surname@di.unito.it).
} 
The presence of a multiplicity of (possibly heterogeneous) UAS requires innovative solutions in the global control architecture (see, e.g., [2]) since a number of problems have to be handled:

- How to distribute tasks among UASs in order to make the best use of the capabilities of the UASs (and of the sensors) and to avoid interferences among the UASs.

- What kind of co-operation is needed between the UAV and the operators of the ground control station.

- What kind of situation awareness can be obtained by the human operator.

Some recent projects have addressed the problem of co-operation by studying both centralized and decentralized approaches, with a significant emphasis on increasing the level of autonomy of the UAS (see. e.g.. [3-8]).

The results obtained in these projects are promising and show the potential of co-operation in solving complex tasks. However, in most cases the investigation has been carried on with prototypical vehicles and the operational scenario was simulated.

The approach adopted in SMAT-F1 is innovative for several reasons:

- The covered area was the real area of operations of a territory surveillance system for Regione Piemonte, over a surface of more than $1500 \mathrm{~km}^{2}$. The overflown area was populated and the flight permissions had to be requested respecting all the rules and constraints of flight in civil air space.

- The surveillance mission targets were selected in order to include typical situations of real operative scenarios, such as pollution detection, industrial plants, traffic or landslides monitoring, river monitoring along valleys in case of floods, monitoring on specific mountain zones (fire detection).

- The UAVs involved in SMAT-F1 program belong to three different classes: MALE = Medium Altitude Long Endurance (Sky-Y by Alenia Aermacchi); MAME = Medium Altitude Medium Endurance (Falco by Selex Galileo); and LALE Low Altitude Low Endurance (C-Fly by Nimbus). Therefore all the UAVs are Italian industrial products.

- The sensors were commercial products used in UAS for both military and civil applications.

The previous choices allowed us to make a novel and significant experience on all the phases of real mission preparation, execution and coordination, with a particular attention to all the aspects of interaction between the system and the human operators.

The project described in this paper was focused on the demonstration of the integration of the flying platforms with the Supervision and Coordination Station (SSC), designed with the purpose of collecting and processing data coming from the UASs. The demonstration was carried out performing an in-flight test campaign in an area located in Piemonte involving the 3 UASs coordinated by the SSC.

The final demonstration was a proof of the effectiveness of Mission Planning design and of the system capabilities in collecting, displaying and efficiently archiving mission data, as well as a source of suggestions for future work.

\section{OPERATIONAL SCENARIO}

The operational scenario of the multi-UAS mission for SMAT-F1 involves many components (the main ones are shown in Figure 1): 


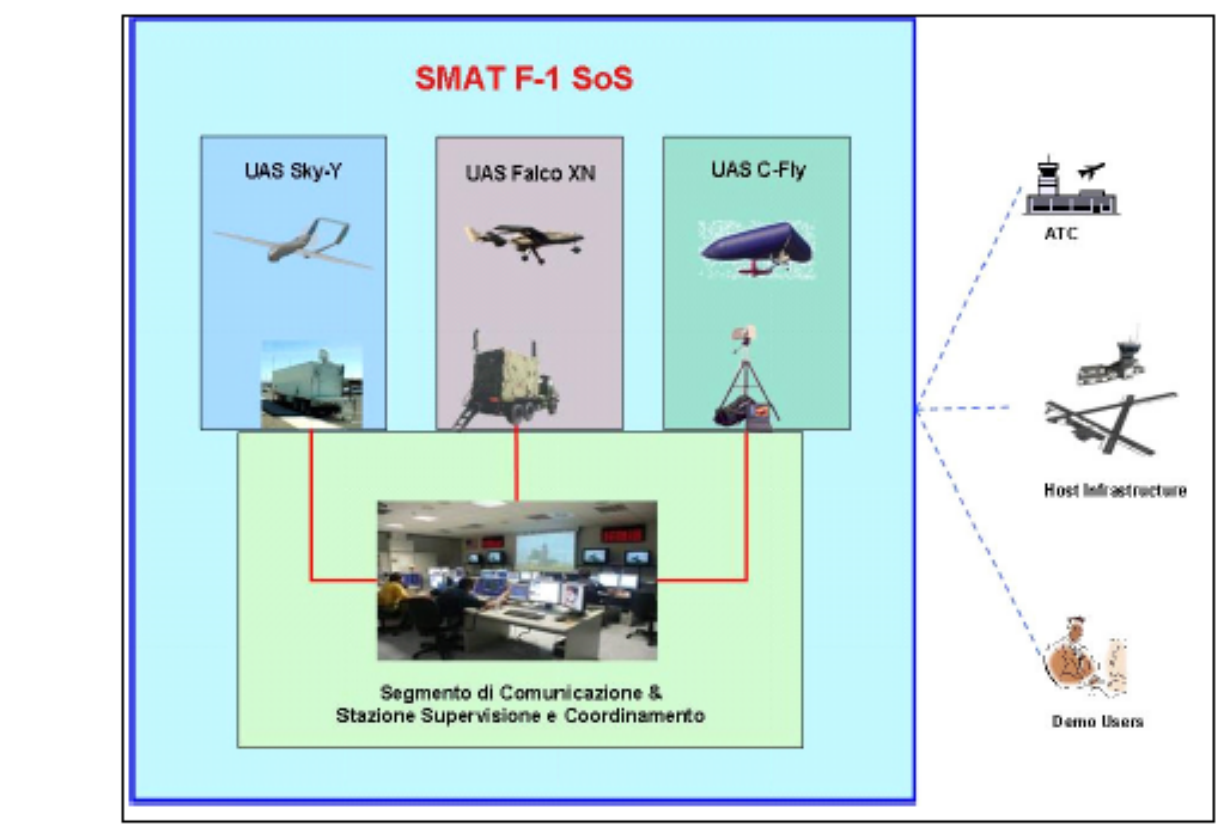

Figure 1.

Operation scenario

- The UAVs that execute the mission over an Area of Interest, each equipped with the necessary sensors specific for the requested monitoring mission.

- The Control Stations where the operators plan the flight, pilot the UAV and control the sensors. The CS is responsible for the safety of the flight and the coordination with air traffic control (ATC) entities. It receives the mission plan from the SSC for final approval and executes the planned mission.

- The SSC (Stazione di Supervisione e Coordinamento, i.e., Supervision and Coordination Station) where the operators plan the overall integrated mission based on end-user requirements, negotiate/coordinate the mission with the CSs, follow and coordinate its execution, store and analyze data, distribute the results to the end-users.

- The data communications network (Segmento di Comunicazione) is the interconnection network between each CS and the SSC for data, voice and video exchange.

The three UAVs were controlled by the three UAV Control Stations (CS), used to control the air-vehicles and relevant payloads.

All three UAVs were equipped with optical sensors. Sky-Y and Falco mounted a Main Turret with optical/IR sensors; Falco mounted, in addition, the Selex Galileo SAR radar PicoSAR.

C-fly was equipped with a TV camera and a special chemical substances detector.

The CSs of Sky-Y and Falco were connected to the UAVs via a redundant data link (NBDL and WBDL) for command and control of the aircraft and payloads. The C-Fly had only one data link.

Table I summarizes the main characteristics of three UAVs.

All CSs are individually connected to the SSC through a dedicated network for exchanging planning data, send real-time telemetry and sensors data, allow voice coordination for the operations (using a SIP based VoIP application), and exchange additional flight recorded data not transmitted in real-time (see Figure 2). 
TABLE I. UAVS CHARACTERISTICS

\begin{tabular}{|l|c|c|c|}
\hline \multicolumn{1}{|c|}{ Characteristics } & \multicolumn{2}{c|}{ UAVs } \\
\cline { 2 - 4 } & Sky- $\boldsymbol{Y}$ & Falco & up to 2 \\
\hline Operational Altitude [kft] & up to 20 & 18 & max 45 \\
\hline Speed [kts] & $\max 150$ & $\max 134$ & 6 \\
\hline Persistence [h] & 14 & 14 & $<5$ \\
\hline Payload [kg] & 150 & 70 & max 15 \\
\hline Range datalink $[\mathrm{km}]$ & LOS radius $>130$ & max 200 & EO/IR, chemical \\
\hline Sensor suite & EO/IR & SAR, EO/IR & \\
\hline
\end{tabular}

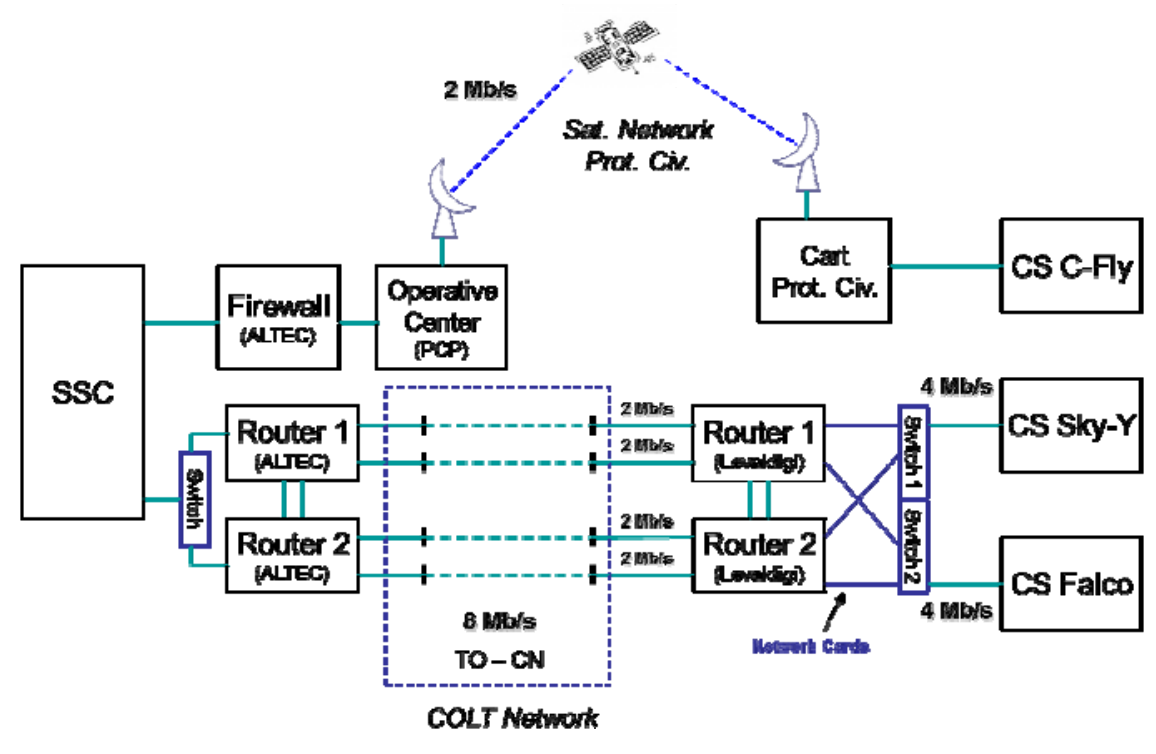

Figure 2 .

SC\&SSC Network System Architecture

For the flight test of the project and the final demonstration flight the Area of Interest for the mission was identified in a wide area covering the south-west part of the Piedmont region, part on the Alps and part on the plains of the Cuneo province. The selected area was identified after a joint study and approved by the Italian Civil Aviation that anyway imposed some flight constraints due to the current lack of flight rules for UAVs (of the same type of the ones employed in SMAT-F1) over an inhabited area.

For logistic reasons, the CSs of Sky-Y and Falco were both based at the Cuneo Levaldigi airport, and this allowed to connect both CSs to the SSC via a dedicated terrestrial link with an overall aggregated bandwidth of 8 Mbps (four E1 Links, 2 Mbps each).

The C-Fly was deployed in a small airport near the Levaldigi area (Benevagienna) and point-to-point connectivity with the SSC was obtained via a 1 Mbps Skyplexnet satellite link (made available by the Piedmont Region Civil Protection that has one terminal at the ALTEC facilities).

\section{SSC}

The SSC, installed at the ALTEC center in Turin, is the terminal concentrator of all network connections of the different CSs (it is worth to note that there is no direct interface among the CSs).

From an HW point of view, the core of the SSC data system is represented by a cluster of servers that run the SSC SW, connected to a Storage Area Network making available to the system around 4 TB of disk space.

The SSC SW (see later on for more details) provides the following main functions: 
- Provides support to system configuration, mission definition and activities planning, including automatic export of the mission plans and import of the flight plans to/from the CSs.

- Receives real-time telemetry and sensor data, processes them in real time, stores them in the DB and distributes them to the client computers for analysis and display to the operator.

- Provides support for the post-mission data processing and end-user products generation.

- Provides a database designed in order to save mission products and to support the users in postmission data retrieval and processing. External data import for mission support is allowed.

The operator consoles of the system are located in the SSC operations room (see Figure 3).

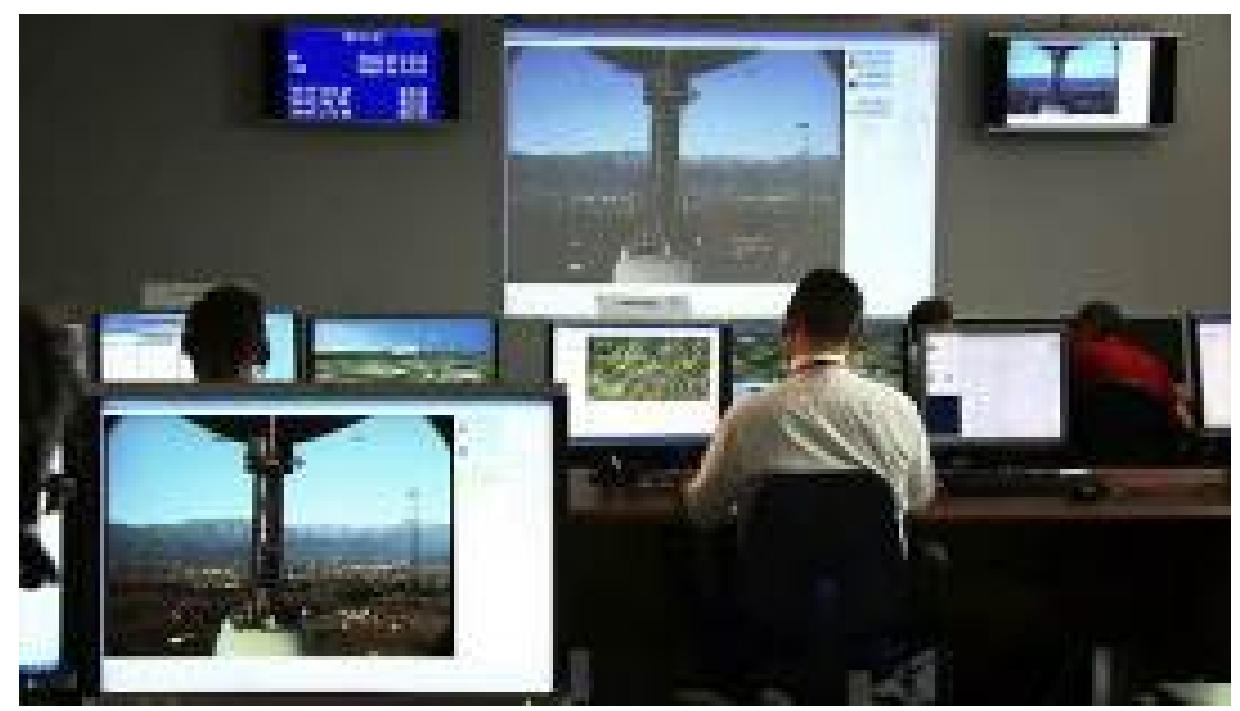

Figure 3.

SSC room configuration

Each operator workplace is equipped with two consoles:

- One dedicated to the execution of the client portion of the SSC SW, i.e. to planning, real time monitoring and data analysis, and data post-processing (see Figure 4).

- The other dedicated to operations support tools (like the mission log for events recording and problem tracking) and the voice coordination system (see Figure 5).

The room is equipped with a projector allowing to share relevant images with all operators for mission status awareness, and a time display, providing time support information to the operators (current time, time to event $\mathrm{A}$, time to event $\mathrm{B}$, etc.). 

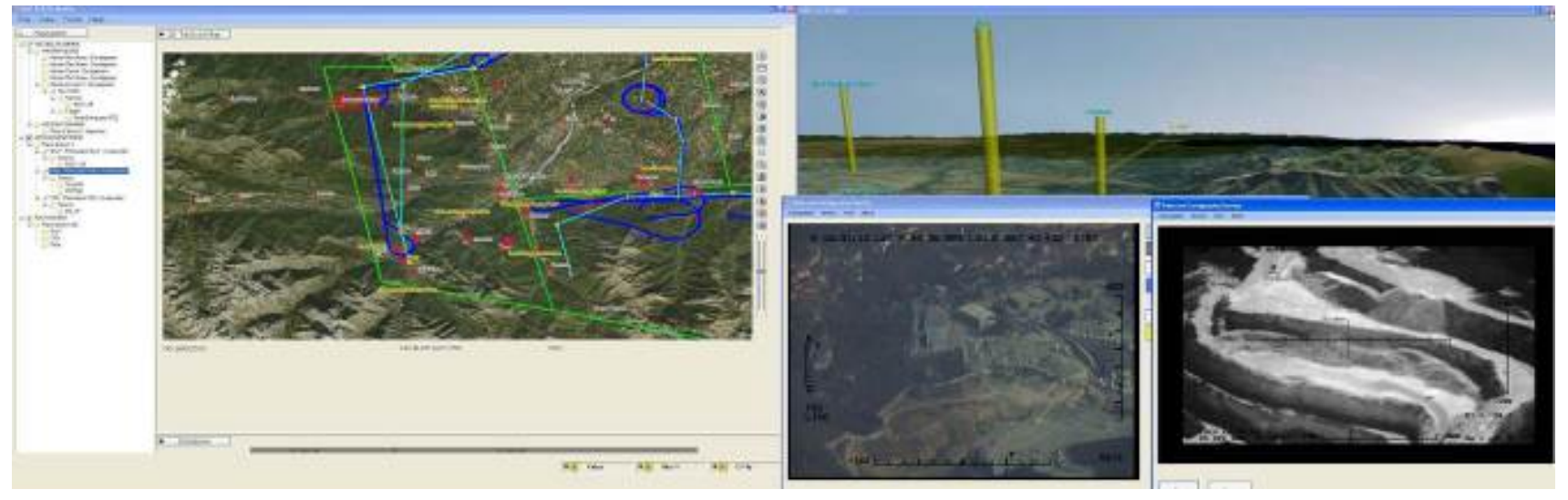

Figure 4.

SSC workstation

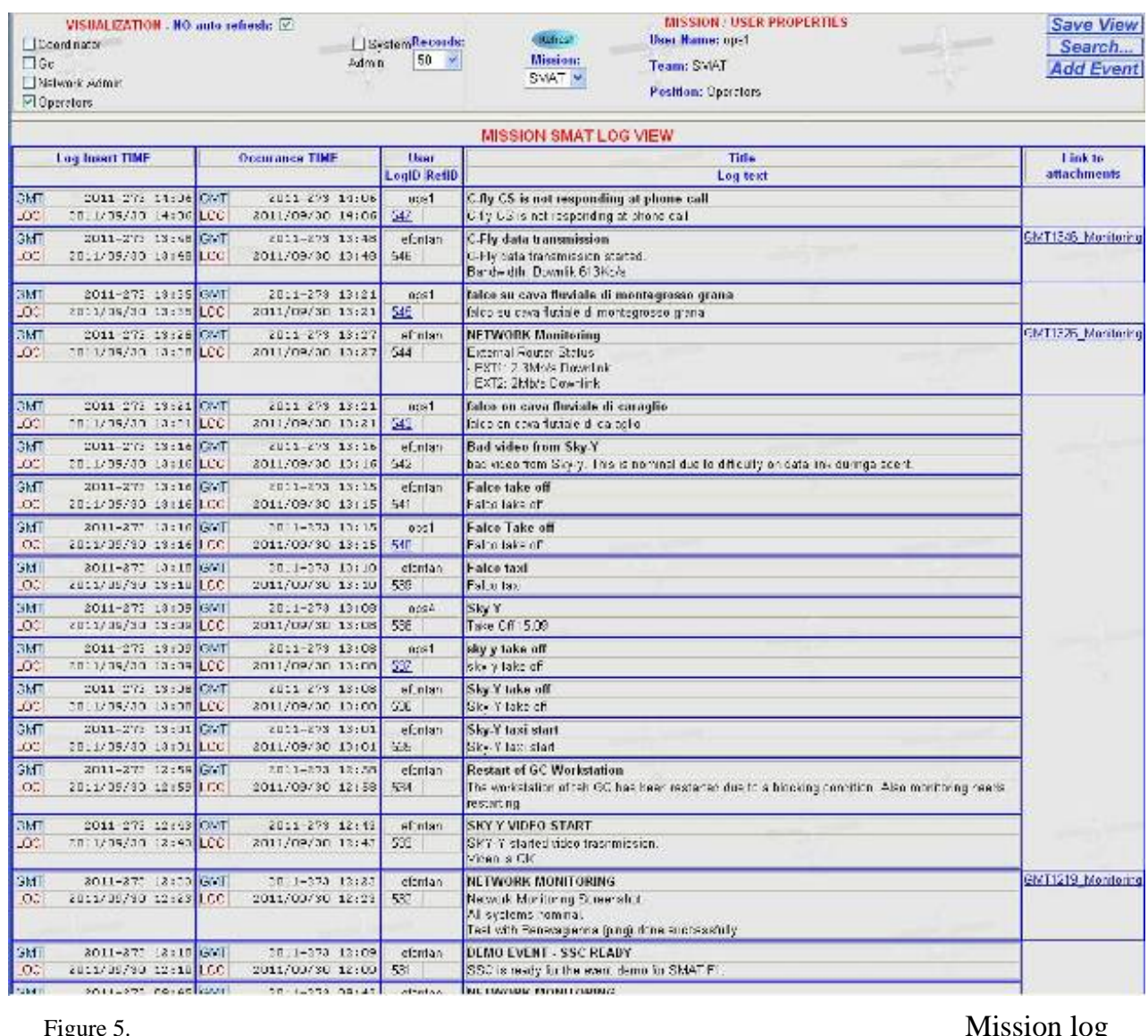

\section{HUMAN OPERATORS ROLES}

A fundamental aspect to be taken into account in this complex scenario are the roles and responsibilities of the human actors.

The CSs are normally managed by two operators: 
- The pilot is responsible for the plan to be flown; he receives the Mission Plan from the SSC and has the capability to refine this high level Mission Plan according to the UAS platform constraints and send the resulting flight plan back to the SSC.

- The sensor operator has the responsibility to command and control the payloads during the mission.

The SSC team has different operators:

- A Ground Controller who controls the SSC data systems and the communication network.

- An Operation Coordinator (OC) who has the mission planning and execution coordination responsibility.

- Three Single Operators (SO) who are responsible for single UAV mission planning and mission following, as well as for any data analysis and processing.

As already highlighted, the SSC is the only element of the system that has the overall view and responsibility of the integrated mission of the different UAVs. The Operation Coordinator is responsible for giving appropriate instructions to reach the mission objectives, taking into account also unplanned events.

The preparation and execution of an integrated mission in the SMAT-F1 system is constituted by the following phases:

- Mission Design: the Operation Coordinator receives the Mission objectives from an external source (i.e. the end-user) and, based on the required type of monitoring activity and the involved area of the territory, assigns to the mission the UAVs, the targets to be observed and the sensors to be used.

- Mission Planning: The Mission Plans for single UAVs and the overall Mission Plan are then developed in more detail by OC and SOs. After final approval by OC, each UAV plan is sent to the corresponding CS that requests the ATC approval. If necessary, the plan can be modified (in an iterative way) in order to take into account, for example, additional constraints imposed by environmental conditions or by the status of the selected vehicle.

- Mission Execution: the mission is then executed. The CSs implement the agreed plan while the SSC performs mission monitoring and coordination. Moreover, the SSC performs in real time the initial data processing on all received data.

- Post-mission analysis: when the mission is completed, the SSC, if necessary, receives additional flight recorded data. Stored data are post-processed based on the type of output to be provided to the end user.

The following sections will describe in detail the first three activities, while a detailed description of Postmission analysis is out of the scope of the paper. It is just worth mentioning that Post-mission capabilities include advanced retrieval functions and image processing algorithms (such as smoothing, contrast enhancement, etc...).

Various displaying capabilities have been developed (for example Superimposition of geo-referenced images on maps with transparency setting, including SAR generated images). In order to better contextualize the data, a data mining tool (called MetaDataRetrieval) has been developed. This module is able to automatically extract relevant (geographic and social metadata) information concerning the area of interest from publicly available sources on the WEB (e.g., Geonames and OpenStreetMap), see [9, 10] for details. 


\section{TASK MODEL}

During the system design phase, one of the main activities was to define the entities constituting a Mission and to define the set of relationships between such entities.

The Mission had to be a container of entities suited to:

- Allocate resources to the mission: platforms, sensors, targets assignments.

- Plan flights: routes, waypoints.

- Plan sensors usage: define mission phases (tasks) along the route to cover targets.

- Collect all mission products.

In particular, the Mission was structured as a hierarchy of entities easily accessible by operators in all phases: planning, mission execution and post-mission analysis.

The following scheme shows the logical hierarchy for Mission Planning:

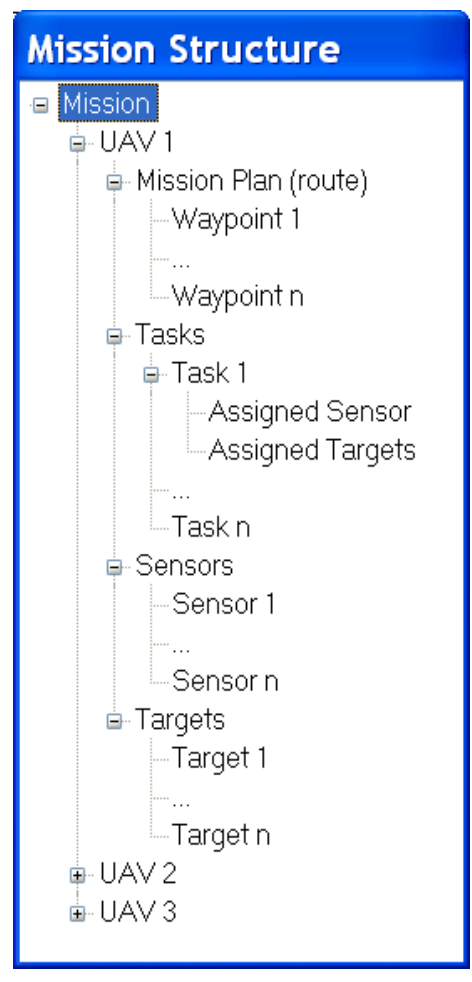

Figure 6.

Mission Planning Structure

The main entity is the Mission. Up to three platforms can be assigned to a Mission. Targets to be covered and sensors to be used can be assigned to each platform. After this, a route constituted by waypoints is defined for each platform. Once a route has been built, it is possible to assign a task for each piece of route, where a "piece of route" is defined as one or more route "legs" delimited by route waypoints. Specific sensors and targets are allocated to each task. The task structure is quite rich since several pieces of information are associated with the task and the waypoints (i.e.: expected altitude, speed and heading are associated with each way point).

This planning structure is reflected, for the user, into a Mission Tree. The plan is displayed on the map and the tasks are depicted in a Scheduler widget, showing the Task sequences along a mission timeline (see figures 7 and 8): in this way, the operator can get the spatial planning information from the map and the temporal one from the scheduler. 
Using the Mission Tree the user can access nested information. The Mission Tree is subdivided into three sections, corresponding to the main states of a Mission:

- Tactical Planning, with the design and planning sub-phases, as explained below.

- Mission Monitoring, active during flight.

- Post Mission, active for completed missions.

The described hierarchy is accessible in different modes by the user, according to the three phases:

- In Tactical Planning all the items are accessible although there are obviously no mission generated products attached.

- In Mission Execution the Plan is in read-only mode and, by selecting a specific sensor, the data/images/video generated by that sensor can be displayed.

- In Post-mission, for each sensor it is also possible to display the results of post-processing (for example, the mosaiking generated from images acquired during the mission).

\section{MISSION DESIGN AND PLANNING PROCESS}

Mission design involves the assignment of UAVs, targets and sensors to the mission. In this demonstration phase the assignment was made allowing the operator to select information from existing stored data. The final result is a preliminary sketch of the mission structure. Such a mission structure is made available to the Mission Operators that build a Mission Plan for each selected UAV.

The characteristic of Mission Planning in the SSC is that its main purpose is not the generation of a flight plan, but the correct assignment and configuration of sensors along a route, where the route is drawn to cover in the best way the targets assigned to the mission; each target may be a point, a linear object (river, road), or an area.

In a multi-UAV scenario this goal is more complex, because the mission optimization has to be achieved combining in the best way the plans characteristics and sensor usage of all UAVs without conflicts.

The planning interface of human operators is reported in Figure 7. The Mission Planning function supports two different user levels, OC (Operation Coordinator) and SO (Single Operator).

In particular, each SO builds a raw Mission plan for the UAV assigned to him, taking into account the platform configuration and targets assigned to the UAV. It is worth noting that Mission planning is not just route drawing. The complete Mission planning has to take into account also sensor planning, the description of the desired behavior of the sensors along the route. The final result of Mission planning by each SO is a Mission plan which maps a sequence of tasks (including observation tasks) to the route.

Tasks information is displayed (both to SOs and OC) on a scheduler timeline to highlight mission flow (Figure 8).

When a SO is building a Mission plan, the OC is not allowed to modify the plan until the SO is ready to deliver it. The SO, before delivering a plan, can execute an automated validity check (see next section).

The OC analyzes the delivered Mission plans by taking into account different constraints of the mission (time, distance, sensor coverage constraints). In particular, he examines the planned route of each Mission plan and the related scheduled times, and decides whether the targets are covered and observed at the right time. 

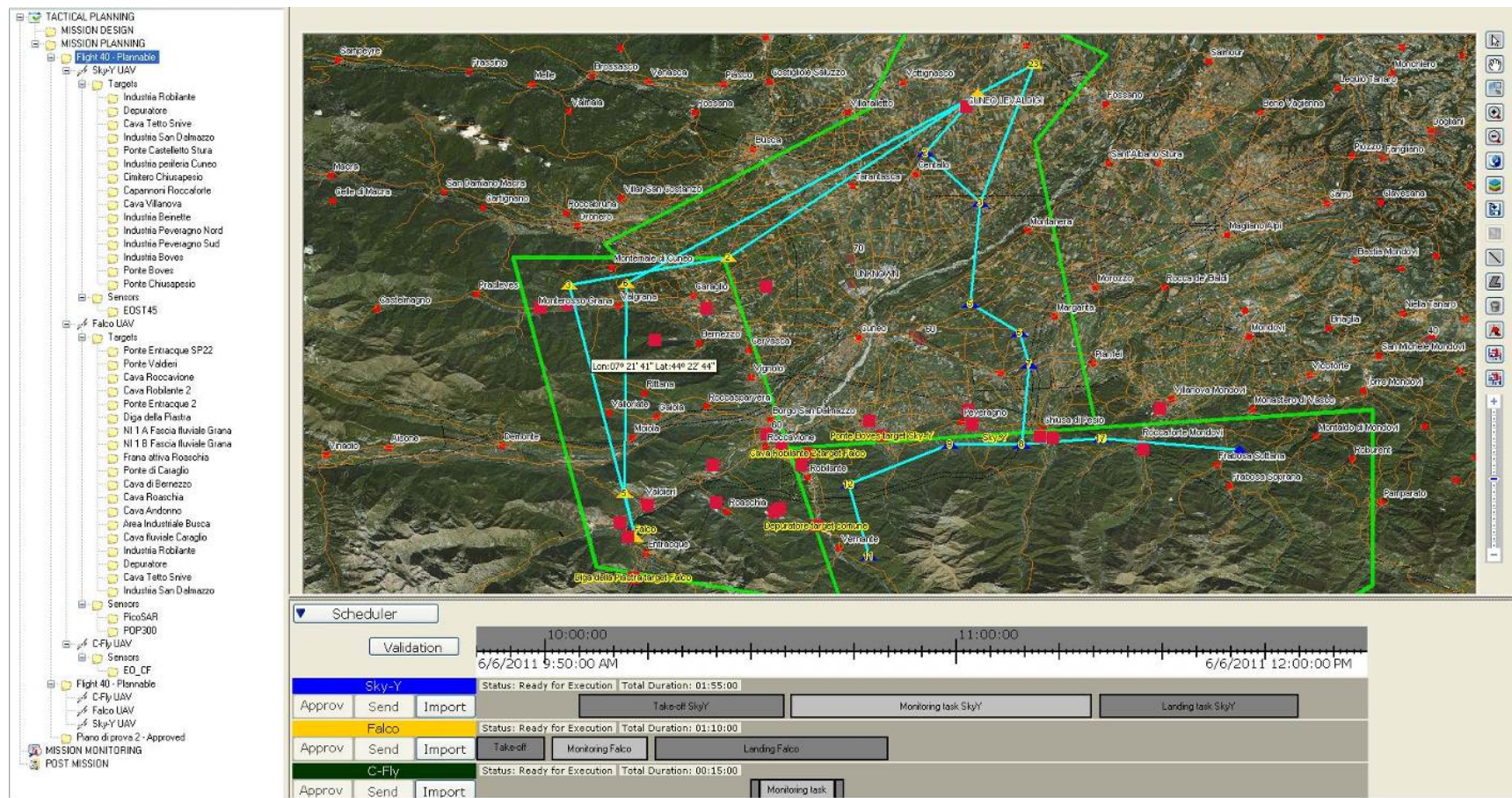

Figure 7.

Mission Planning Interface

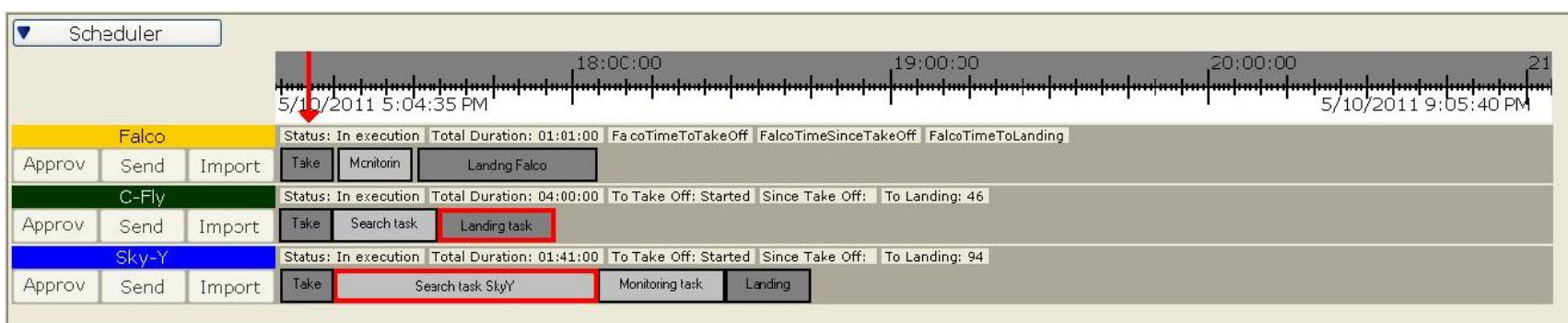

Figure 8.

Scheduler

This process can require several iterations between the OC and the SOs to refine the plans. When all single plans are approved, the OC is able to validate the overall plan (again, using the automated validity checks described in the next section).

The final step is the delivery of Mission plans to the CSs for the final formal approval of each single UAV Mission plan. Also in this step, it is possible to iterate several times between the SSC and the CSs, until each plan is finally approved and the process is completed.

During these iterations, the OC constantly revises the three (current) Mission plans to check that they cover the mission goals correctly both in terms of spatial and time constraints.

\section{DECISIONAL SUPPORT TOOLS}

An Intelligent Assistant (IA) has been developed for supporting the operators during the planning process in checking the validity of the mission plans. The usefulness of such a kind of tools is being increasingly recognized in both the UAS and the space missions domains (see e.g. [11, 12]).

The first goal of the IA is to automatically check whether the mission plans under examination meet a set of mission requirements and domain constraints. Moreover, in case of violations, the IA has to single out the parameters that are possible causes of such violations. Finally, in some cases the IA is also able to 
suggest how to change some parameters so that the violated constraint(s) become satisfied. Please see [13] for more details.

The IA reasoning capabilities are based on the availability of several kinds of knowledge and information:

- physical and operative characteristics about UAVs and sensors (stored in database); typical examples are the max speed and max fuel capacity of a UAV, and the max resolution or FOV (Field of View) of a sensor.

- models for estimating parameters that are not explicitly given in the mission plan, but are needed for checking its validity; typical examples are the estimation of the fuel consumption given the length of the route and the kind of UAV, and the coverage of a target given the altitude of the UAV and the FOV of the sensor.

- mission constraints which restrict the contents of the mission plan by also taking into account the mission requirements; typical examples are the check that each leg of the route belongs to exactly one task, and that the estimated duration of the mission falls within the specified time envelope.

It is worth noting that some constraints concern the mission plan of a single UAV, while other constraints involve several mission plans. The sample mission constraints mentioned above refer to a single mission plan. Instead, the check that all of the mission targets have been assigned to at least one UAV is a typical check involving more than one mission plan; another example is the check that a target Trg1 assigned to a UAV is observed before a target Trg2 assigned to another UAV as specified.

Constraints are encoded as rules in the CLIPS rule-based system (http://sourceforge.net/projects/cliprules), which is able to perform different kinds of inferences thanks to different kinds of rules:

- rules for detecting constraints violations.

- rules for singling out possible causes of violations.

- rules for suggesting changes of parameters to fix the violations.

While the detection of violations and the tracking of the causes of the violations in terms of the involved parameters is always performed, the suggestion of ways to repair the plan is possible only in some cases, while in other cases this is left completely to the user.

The main advantages of encoding constraints into rules instead of hard-coding them into the software are the following:

- rules represent templates of constraints that are automatically instantiated. For example, a rule that says that the velocity of a certain kind UAV at a waypoint should not exceed the maximum speed of the UAV is automatically applied to all of the waypoints of the route by the matching mechanism of the rule-based system.

- rules encode constraints in a declarative form and therefore it is much easier to determine which constraints are applied and how they are applied in a flexible way, depending on the mission context.

- rules decouple inference from complex computations, that can be embedded as simple predicates and functions that appear in the rules (e.g. geometric reasoning for target coverage, temporal reasoning for durations).

The second point above is particular advantageous for civil missions, where constraints are often strict but can vary according to context, e.g. they can differ for different kinds of areas (populated areas, industrial areas...). 
To conclude this section, let us consider a sample of output from the Decisional Support module (see Figure 9).

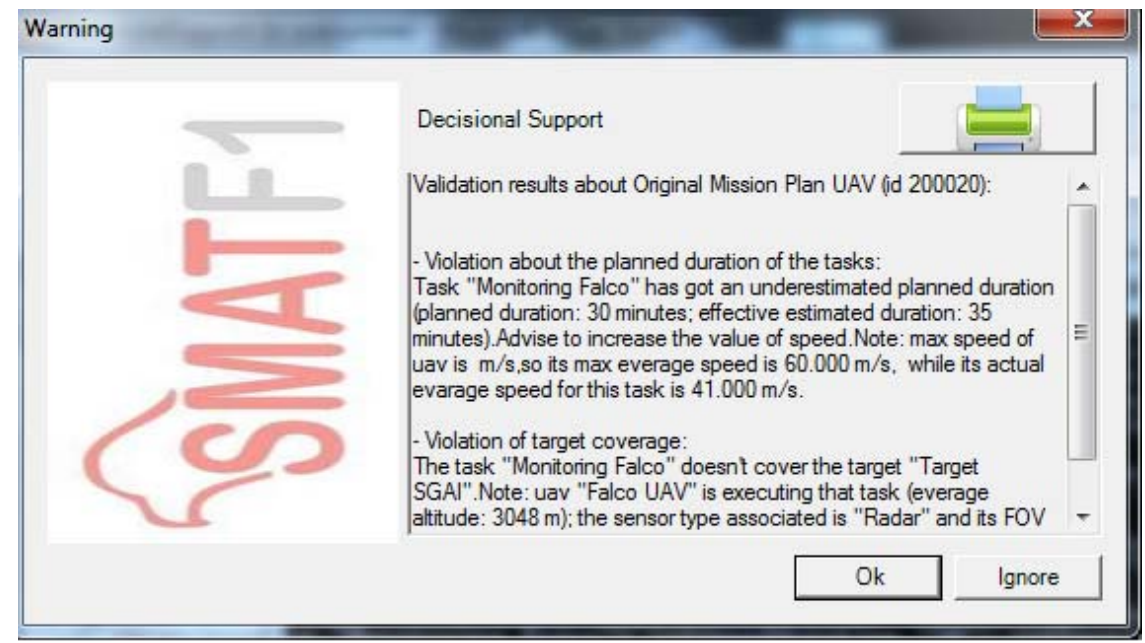

Figure 9.

Sample Output from the Decisional Support

The first message reports a violation of the constraint about the duration of the Mission Plan, since the estimated duration of the mission is $35 \mathrm{~min}$ while the time envelope for the mission is just $30 \mathrm{~min}$ (violation detection). The parameter which causes the violation is the average speed of the UAV, which in the current plan is $41 \mathrm{~m} / \mathrm{sec}$ (cause of the violation). Taking into account the fact that the maximum average speed of the considered UAV is $60 \mathrm{~m} / \mathrm{sec}$, the Decisional Support module suggests to increase the speed of the UAV (repair suggestion). The figure also shows part of a second message, concerning the covering of a target and the relevant parameters (UAV altitude and sensor FOV) that cause the failure to cover such a target.

\section{MISSION EXECUTION SCENARIO}

The developed system was tested during a sequence of flight tests, at first with a single platform and then with all the platforms involved. The SMAT-F1 project was completed with a final public demonstration held on 30th September 2011.

During these activities we have tested on the field the system's capabilities versus the real mission operational needs. These tests and demonstrations constitute the first example of multi-UAV mission flight on civil populated areas in Europe for this type of UAVs. The experience made is therefore a unique source of information for the analysis of the system capabilities used for the human mission coordination.

The mission of the demonstration was designed to cover three specific areas: two less populated areas on the mountains to be covered by MALE and MAME UAVs (Sky Y and Falco), and a small plain area assigned to the LALE UAV C-Fly.

It was planned to cover targets both during the initial flight phase (to check sensors behavior) and in the areas on the mountains selected as specific mission target areas.

Mission paths and targets were chosen to test information acquisition tasks typical of civil surveillance and monitoring missions. In particular the targets were (see Figure 10): 


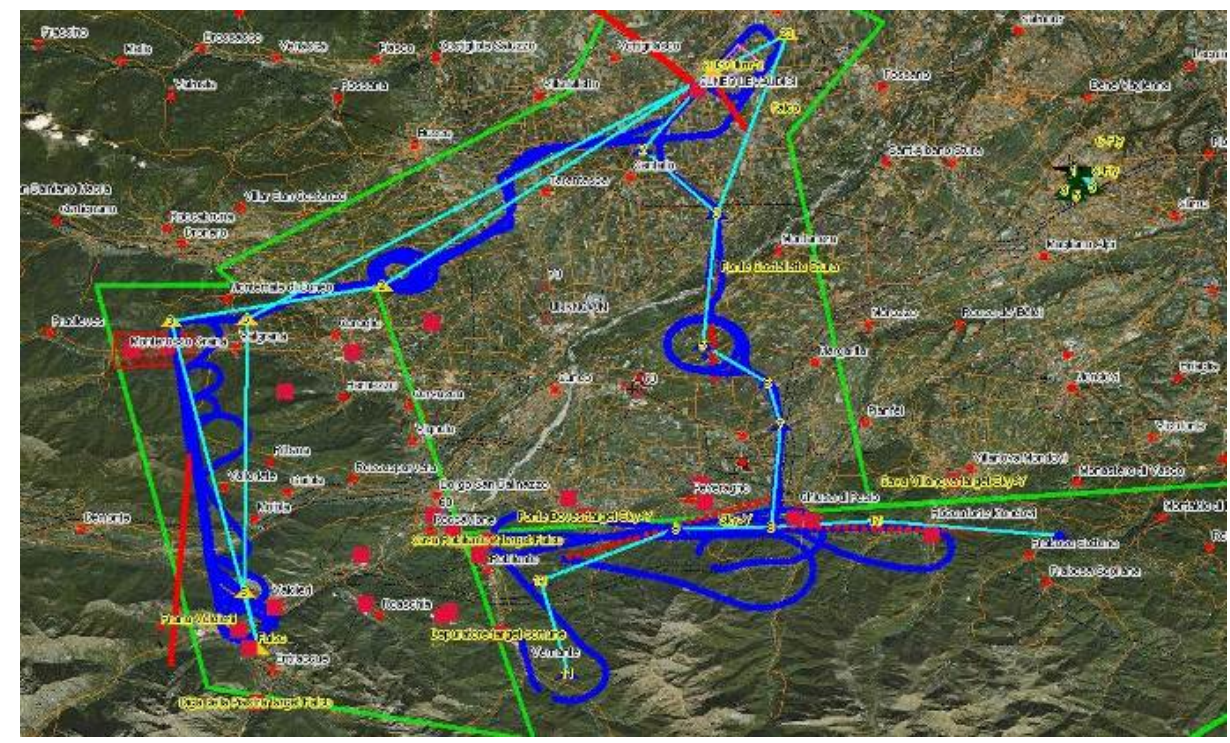

Figure 10 .

Targets Area

- Industrial plants, to be monitored both in case of floods and plant malfunctions or pollution.

- Sensible points on motorways or roads (bridges) to be monitored as a traffic control routine task or in case of calamities.

- Zones identified as critical in case of flood due to meteorological events, i.e., sequence of bridges and floodable areas along valleys.

- Mountain zones with quarries, chosen to stress the capability to monitor localized events (i.e., to monitor fires and landslides...) during flight on mountain zones.

After the mission start, all the operators (both SOs and OC) were provided with the capability to display on their own workstations the following information (see Figure 11):

- Planned vs. flown route on 2D map.

- Flown route on 3D map from customizable points of view.

- Time of flight along the planned tasks scheduling timeline.

- Video display from sensors with cartographic overlay capability.

- Tabular display of navigation and sensor data.

- Access to images and videos from past missions.

To evaluate the system capabilities, let us consider in deeper detail the roles of the operators at the SSC during Mission Execution.

The overall goal of the SSC team was to get from the Mission all the relevant information in the most efficient way. To achieve this goal it was necessary to have a very clear picture of the Mission status from the system. In addition, the team could interact with the CSs operators by voice, not only to get information but to suggest specific actions. To fulfill this task a strict subdivision of responsibility between the OC and the SOs was needed:

- Each SO follows his assigned platform flight on his own workstation. He communicates only with the assigned CS and with the OC. 


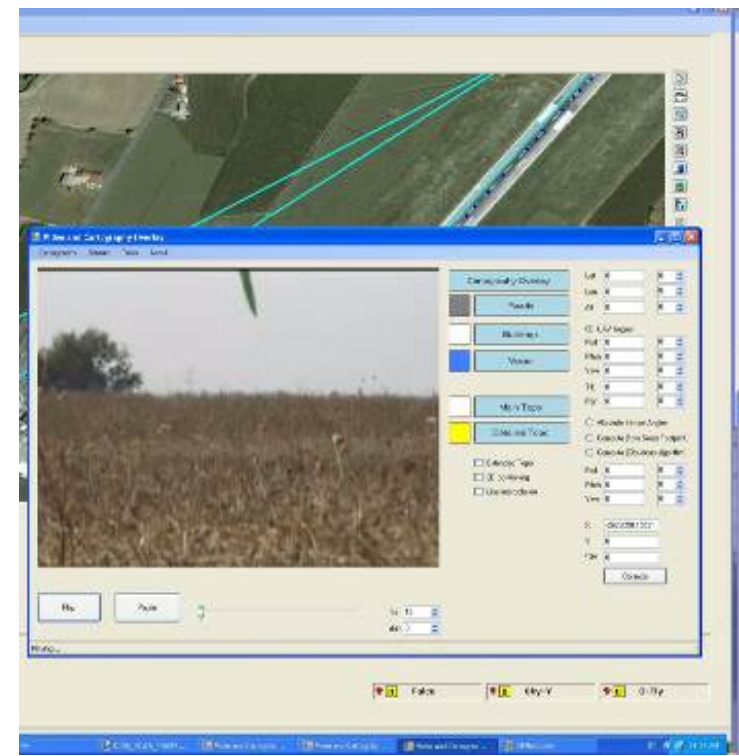

Figure 11.

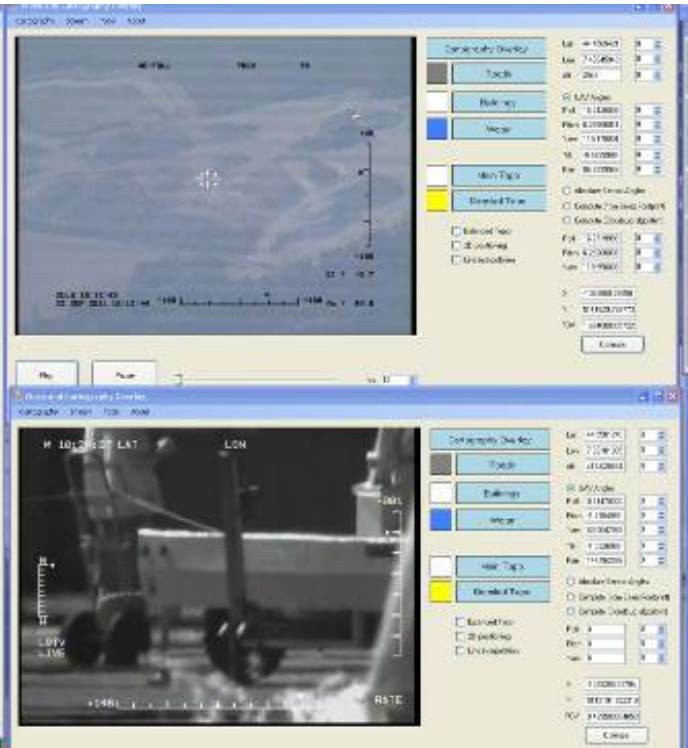

Displays

- The OC follows the three flights and communicates with the CSs only via the three SOs.

These two rules prevented confusion in information request and exchange.

Each SO must check that the targets assigned to the single platform are covered and the data concerning the targets are correctly received by the SSC.

The OC main goal is to get a clear understanding of the correct evolution of the entire mission, because he is the only actor that can have the knowledge about all the three flights being executed. He must intervene to support CSs for any problem of single mission misalignment, and he has the specific task to drive CSs operation during joint actions in target areas, paying particular attention to targets sequence and observation of the same target.

\section{MISSION EXECUTION DEMONSTRATION}

Let's examine the typical execution of a mission to highlight the operational results of the interaction between the operators and the system.

The longer missions were the MALE/MAME ones. The two missions started at slightly different times (from the same airport), then the UAVs executed loiter manoeuvers to reach the desired surveillance altitude. During this preliminary phase the operators task was to check the communications and the UAV information displays.

Then, a specific target (different for each UAV) was pointed to have a first test of video sensors usage. The operators checked via voice the start of the planned target acquisition and prepared for the video display. In addition, they tested the retrieval facility for getting from the database images of the target got during previous missions. This facility, in general, is useful both for target correct identification in case of weather problems and for detecting changes in the target zone characteristics.

When the platforms entered the surveillance zone, a valley was followed and floodable zones monitored. In this case each SO had to check the correct monitoring of different zones and he could possibly request non-planned zooming actions in case of events of interest (river occlusions...). The system displayed on the 2D map, in near real time, the sensor coverage and, if this was not sufficient, the operator could check on the 3D synthetic representation the sensor field of view from the platform position (see Figure 12). 


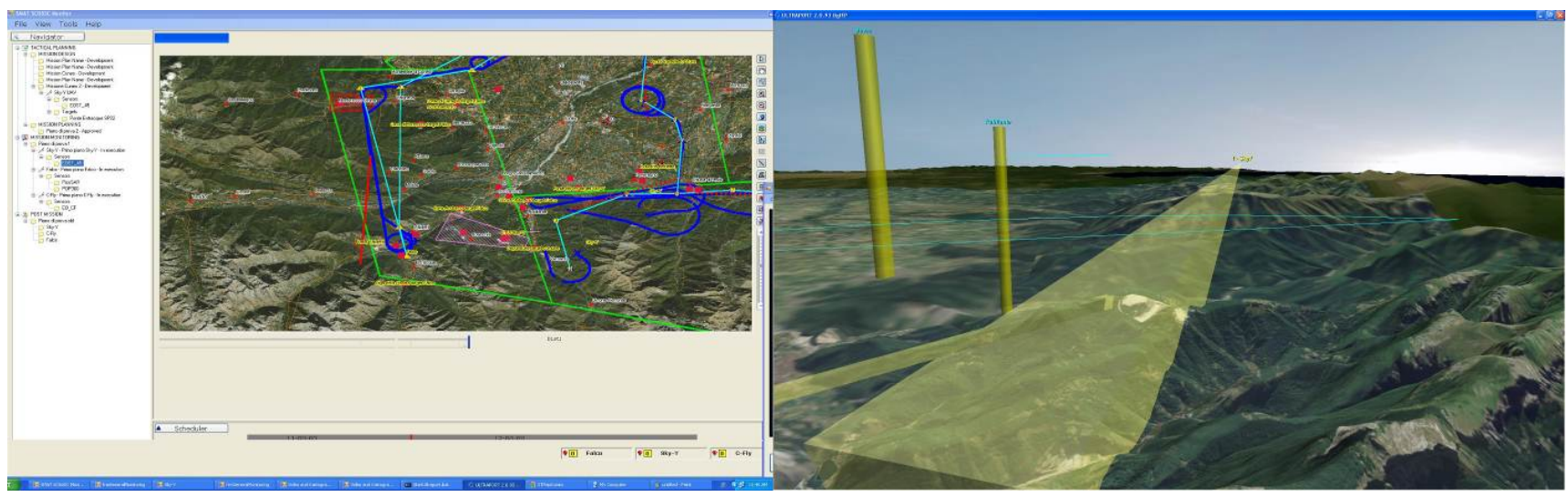

Figure 12.

2D and 3D coverage (the latest provided by

Synarea)

The SO following the C-Fly mini-UAV received from the CS the ready to start for its mission and checked with the OC if the overall mission was on time or if take-off delay was needed (this flexibility was possible because the mini-UAV is less constrained on take-off time). The OC checked the current situation both on his display and by voice with the other SOs to verify that no critical target acquisition was on execution and then told the C-Fly could start.

With all platforms on flight, the OC checked the correctness of the joint display of all the three routes, the three videos and related data tabular displays on his two screens.

During next target overfly, the SOs not only watched the videos, but also took some snapshots to archive specific views. Their work was supported by real time superimposition on videos of synthetic cartography displaying features as toponyms, roads and rivers.

In the southern part of the Piedmont the target areas assigned to both MALE and MAME were adjacent and the mission involved also the simultaneous surveillance of a common critical planned target; in particular, the two platforms covered with the optical sensors the same target from different positions, one in optical mode and the other one in IR mode.

In this phase the role of the OC was important: he had to check on the displays the correctness of the platforms operation, to verify the mission time on the scheduler, and to check via the SOs the situation on the CSs.

To have the complete view of the mission, the OC could set the 3D display in Free Fly mode to see the two platforms and their fields of view simultaneously from a selected point of view.

When the most critical point of simultaneous target monitoring was reached, the OC and the SOs interacted strictly with the CSs to syncronize the activities and to drive the platforms on the target. The manoeuver was fully successful, and simultaneous views of the common target were acquired by video. In Figure 13 the 3D depiction of the two platforms and their fields of view on the target is shown, along with two snapshots got from the sensors. 


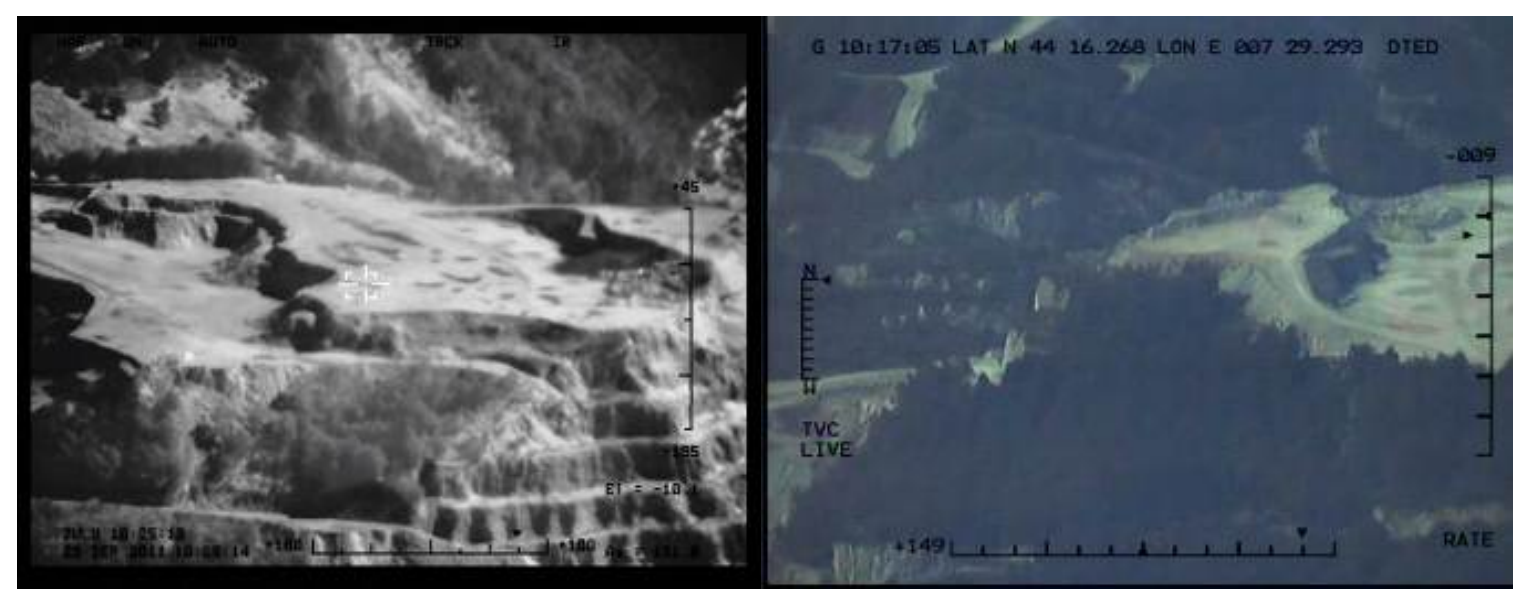

Figure 13.

3D and Common Target

To complete the demonstration of the system capabilities, two interesting experiments were made.

A zone was selected to check SAR radar capabilities. The zone visibility from the planned route was selected accurately to allow to cover the target from the planned flight plan, taking into account that the SAR had to see the target zone under a low angle of incidence. The zone included a place on a hill were several corner reflectors were placed at fixed positions. Corner reflectors maximize radar reflection from chosen points and are used to monitor landslides. Their use makes it possible to detect small terrain displacements using interferometry comparison of two images shot at different times. During the Falco UAV flight the zone was covered once, then the corner reflectors were moved manually to simulate terrain displacement and the zone was covered again. Images were sent back to the SSC, as shown in Figure 14.

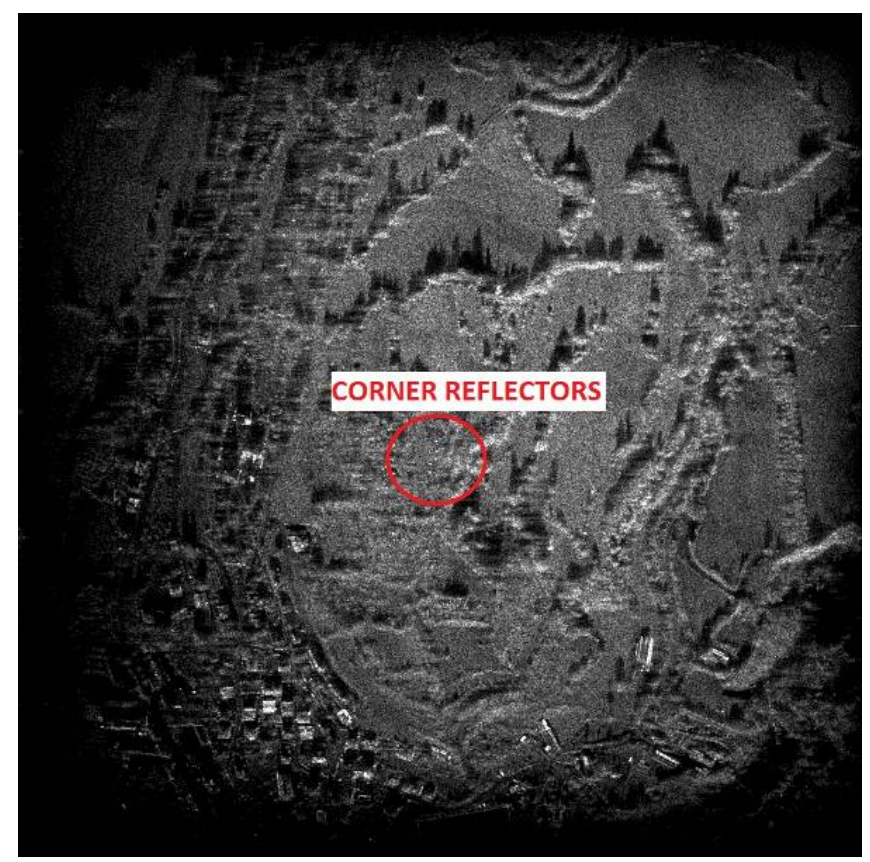

Figure 14. 
The second tested capability was the mosaicking obtained through real time frame extraction and georeferencing from sensor video. A set of selected frames were used to compose a single strip large image of the overflown area. This technique is useful both for normal territorial data acquisition and cartography update and for quick mapping of areas interested by calamities. A mountain woody area was selected and the mosaicking data were saved for post-processing.

The C-Fly mission goal was the recognition of a small area from low altitude (500 meters). In addition, a small quantity of chemical substance was free in the air to allow the chemical detector to save data about its presence.

When all UAVs landed the system labeled the mission as Completed and the mission was moved into the Post Mission section of the Mission Tree.

\section{SCENARIOS FOR IMPROVED PLANNING SUPPORT}

The work done in the SMAT-F1 project has allowed to test the usefulness of automated tools in supporting the human operators. In particular the experience gained in developing the Intelligent Assistant for validating the plans and the availability of a 3D real time visualization facility paves the way for extending the role of automatic support.

For example, the Mission Planning support can be extended to the Mission Design phase, involving sensors assignment by taking into account the UAVs and target characteristics.

Since the task structure is already quite rich and flexible, it easy to extend tasking to provide detailed sensors modes and behavior description along the route. A first kind of support can be obtained by developing an automatic process which inspects the detailed sensor plan and simulates sensor behavior along the route to check target coverage. It is also possible to use a knowledge based approach (similar to the one adopted for automatic plan validation) for "suggesting" sensor modes of operation and behavior to the human operator.

More generally, in the mission design phase, automatic tools could also take into account the temporal constraints implicit in some requirements (e.g., the same target has to be covered simultaneously by more than one UAV) for suggesting to the human operator a correct schedule of the different tasks of the involved UAVs (see, e.g., [14]).

Finally, by taking into account the progress in hardware performance of on-board computers it is becoming possible to move on-board the sensor performance evaluation process and to drive autonomously the sensors during the flight, adapting to the real flight conditions without sensor operator intervention. This would have the benefit of reducing the need of bandwidth from the UAV to the CS and the SSC.

\section{Discussion And Conclusion}

The SMAT-F1 demonstration goals were successfully achieved. The following results were obtained:

- The Integration between the SSC and the single CSs was efficient and allowed quick interaction during mission critical points.

- The SSC Mission Planning structure gave an adequate representation of mission tasking.

- The decision support tools allowed to correct imprecisions and to solve conflicts.

- The SSC capabilities supplied the operators with a clear understanding of the mission during its execution.

- The 2D-3D representation with the display of sensor coverage allowed to drive the sensors in strict interaction with the sensor operators, allowing on time intervention to enhance the quality of acquired data. 
- The role of SSC as a coordination Station was highlighted and the OC was efficiently supported in his role by the system.

Therefore the system developed during SMAT-F1 is mature enough to be adapted to specific needs of Regione Piemonte surveillance system, to be prototyped in the next phase of SMAT.

However, the tested capabilities go beyond this specific application, because they cope with the mission requirements of a wide range of civil applications; therefore, the system supplies a core of functionalities that can be adopted beyond the SMAT program and can be used in general by future civil UAS systems.

The objective of the next phases of SMAT will be the functional demonstration of the whole surveillance system, that will be followed by the design and realization of the system of system prototype and then will be concluded by the industrialization.

\section{ACKNOWLEDGMENT}

The SMAT-F1 consortium has been led by Alenia Aermacchi, and involved Selex Galileo, ALTEC, Politecnico di Torino, Università di Torino, Istituto Superiore Mario Boella, Auconel, Axis, Blue Engineering, DigiSky, Envisens Techonologies, Italia Design Torino, Nautilus, Nimbus, SEPA, SPAIC, Synarea.

The authors are indebted to the practitioners and researchers of all the involved SMAT-F1 consortium partners that have provided their strong support to achieve the project goals.

\section{REFERENCES}

[1] I. Maza, F. Caballero, J. Capitan, J. R. Martinez-De-Dios, A. Ollero, "Experimental Results in Multi-UAV Coordination for Disaster Management and Civil Security Applications,” Journal of Intelligent \& Robotic Systems, Vol. 61 (1-4), pp. 563-585 (2011).

[2] G. Vachtsevanos, L. Tang, G. Drozeski, L. Gutierrez, "From mission planning to flight control of unmanned aerial vehicles: Strategies and implementation tools,” Annual Reviews in Control, Vol. 29 (1), pp. 101-115 (2005).

[3] P. Fabiani, V. Fuertes, A Piquereau, R. Mampey, F. Teichteil-Konigsbuch, “Autonomous flight and navigation of VTOL UAVs: from autonomy demonstrations to out-of-sight flights,” Aerospace Science and Technology, Vol. 11 (2-3), pp. 183-193 (2007).

[4] A. E. Gil, K. M. Passino, S. Ganapathy, A. Sparks, "Cooperative task scheduling for networked uninhabited air vehicles”, IEEE Transactions on Aerospace and Electronic Systems, Vol. 44 (2), pp. 561-581 (2008).

[5] D. T. Cole, P. Thompson, A. H. Goktogan, S. Sukkarieh, "System Development and Demonstration of a Cooperative UAV Team for Mapping and Tracking,” International Journal of Robotics Research, Vol. 29 (11), pp. 1371-1399 (2010).

[6] I. Maza, K. Kondak, M. Bernard, and A. Ollero, "Multi-UAV cooperation and control for load transportation and deployment”, in Journal of Intelligent and Robotic Systems, Vol. 57(1-4), pp. 417- 449 (2010).

[7] L. De Filippis, G. Guglieri and F. Quagliotti, "Path Planning Strategies for UAVS in 3D Environments," in Journal of Intelligent \& Robotic Systems, Vol. 64 (1-4), pp. 247-264 (2012).

[8] A. Schulte, C. Meitinger, “Cognitive and Cooperative Automation for Manned-unmanned Teaming Missions”, Proc. of the NATO SCI-208 Symposium on Advanced Automation Issues for Supervisory Control in Manned-unmanned Teaming Missions, (2009).

[9] E. Roglia, R.Meo, “A SOA-Based System for Territory Monitoring,” in Geospatial Web Services: Advances in Information Interoperability, Peisheng Zhao and Liping Di (eds.), pp. 426-454, Idea Group Inc., (2010).

[10]R. Meo, E. Roglia, E. Ponassi, "MetaData Retrieval: A Software Prototype for the Annotation of Maps with Social Metadata,” in Lecture Notes in Computer Science, Vol. 6913, pp. 642-645, (2011).

[11] J. L. Bresina, P. H. Morris, “Mixed-Initiative Planning in Space Mission Operations” in AI Magazine Vol. 28 (2), pp. 75-88 (2007).

[12] M. L. Cummings, A. Brzezinski, J. Lee, “Operator Performance and Intelligent Aiding in Unmanned Aerial Vehicle Scheduling” in IEEE Int. Syst. 22, pp. 52-59 (2007).

[13]P. Torasso and G. Torta, "Exploiting Automatic Validation in Human Mission Planning," in Research and Development In Intelligent Systems XXVIII, , Ed. M.Brader et al., Springer, pp. 21-34 (2011).

[14] J. L. Bresina, P. H. Morris, “Explanations and Recommendations for Temporal Inconsistencies”, in Proc. Int. Work. on Planning and Scheduling for Space (2006). 
